\title{
MicroRNA-135a induces prostate cancer cell apoptosis via inhibition of STAT6
}

\author{
BIN XU ${ }^{1,2}$, XIAOMING LU ${ }^{3}$, YUMING ZHAO ${ }^{4}$, CHUNHUI LIU ${ }^{1,2}$, XIAOMING HUANG $^{2}$, \\ SHUQIU CHEN ${ }^{1}$, WEIDONG ZHU ${ }^{1}$, LIHUA ZHANG ${ }^{5}$ and MING CHEN ${ }^{1,2}$ \\ ${ }^{1}$ Department of Urology, School of Medicine, Affiliated Zhongda Hospital of Southeast University; ${ }^{2}$ Surgical Research Center, \\ Institute of Urology, Southeast University Medical School, Nanjing, Jiangsu 210009; ${ }^{3}$ Department of Urology, Affiliated \\ Yancheng Hospital, School of Medicine, Southeast University, Yancheng, Jiangsu 224000; ${ }^{4}$ Department of \\ Urology, The First Hospital of Qinhuangdao, Qinhuangdao, Hebei 066000; ${ }^{5}$ Department of Pathology, \\ Affiliated Zhongda Hospital of Southeast University, Nanjing, Jiangsu 210009, P.R. China
}

Received July 15, 2015; Accepted December 6, 2016

DOI: $10.3892 /$ ol.2018.9791

\begin{abstract}
Clinical management of metastatic prostate cancer remains a challenge. Activation of apoptosis signaling pathways via signal transducer and activator of transcription 6 (STAT6) has been hypothesized to be a therapeutic strategy for patients with metastatic prostate cancer. The ONCOMINE ${ }^{\circledR}$ prostate cancer database and two Gene Expression Omnibus datasets (Gene Series 40026 and 21032) were re-analyzed to determine the expression levels of STAT6 and microRNA (miR)-135a in prostate cancer. The current study investigated the induced overexpression of miR-135a in prostate cancer cell lines to detect its function in prostate cell apoptosis using Hoechst staining and fluorescence-activated cell sorting and examined the expression levels of STAT6 and its DNA binding ability using western blotting and an electrophoretic mobility shift assay. In analysis of the ONCOMINE ${ }^{\circledR}$ database, STAT6 expression levels in prostate cancer tissue were higher compared with those in normal prostate gland tissue and were associated with the overall survival rate and biochemical relapse rate following radical prostatectomy. Additionally, there was an inverse correlation between miR-135a and STAT6 expression levels in prostate cancer cell lines. miR-135a was able to induce prostate cancer cell apoptosis via targeting STAT6 mRNA and subsequently repressing protein expression and phosphorylation, which also altered the transcriptional factor function of STAT6 through its DNA-binding capabilities. In conclusion, miR-135a may function as a tumor-suppressing miRNA in prostate cancer
\end{abstract}

Correspondence to: Dr Ming Chen, Department of Urology, School of Medicine, Affiliated Zhongda Hospital of Southeast University, 87 Dingjia Bridge Hunan Road, Nanjing, Jiangsu 210009, P.R. China

E-mail: mingchenseu@126.com

Key words: microRNA-135a, prostate cancer, signal transducer and activator of transcription 6 , apoptosis and its anti-oncogenic activity may involve the direct targeting and inhibition of STAT6.

\section{Introduction}

Prostate cancer is one of the most frequent causes of cancer-associated mortality and diagnosed malignancies in the male population. In 2012, 238,590 new patients were diagnosed with prostate cancer and 29,720 mortalities were attributed to the disease in the USA (1). In early-stage prostate cancer, patients may be cured using radical prostatectomy, whereas it remains a challenge to cure patients with metastatic disease. In the early stages, metastatic prostate cancer is sensitive to androgen ablation therapy; however, the majority of cases progress to an androgen-independent stage (2), which is a primary cause for prostate cancer-associated mortality. Although the American Food and Drug Administration have approved several therapeutic agents for the treatment of metastatic prostate cancer, these therapies have not been proven to prolong the survival of patients beyond two years from the start of treatment.

In the majority of cancer types, apoptosis is inhibited in tumor tissue and the activation of apoptosis signaling pathways is a major underlying mechanism by which cytotoxic drugs are able to kill tumor cells (3). Signal transducer and activator of transcription 6 (STAT6) is a member of the STAT family of latent transcription factors and is able to be stimulated using cytokines, hormones and growth factors that are hypothesized to serve an important role in androgen-independence (4). In a previous study (5), the knockdown of STAT6 in prostate cancer cells promoted apoptosis and it was therefore hypothesized that that STAT6 may be a potential therapeutic target for prostate cancer. However, the underlying mechanisms are yet to be elucidated.

The 3' untranslated region (3'UTR) of target transcripts is bound by microRNAs (miRNAs), which regulate gene expression via inhibition of translation or promotion of mRNA degradation. miRNAs are involved in numerous biological processes, including apoptosis. A particular association has been established between the aberrant expression levels of 
miRNAs and the development, progression and chemotherapy resistance of tumors. The expression of a number of miRNAs has been identified to be upregulated in prostate cancer, including miRNA (miR)-21, miR-125b and miR-221/222 (6). By contrast, miR-7 (7), miR-143/145 (8) and miR-146a (9) have been identified as tumor suppressors. miR-135a is an androgen-regulated miRNA, which is associated with prostate cancer cell migration and invasion. Analyses from the Gene Series (GSE40026) dataset demonstrated that miR-135a has reduced expression levels, particularly in androgen-independent cells (10). In the present study, miR-135a functioned as a tumor suppressor in androgen receptor-negative prostate cancer cells by promoting apoptosis via direct targeting of STAT6 mRNA. This inhibited the protein expression and phosphorylation of STAT6 and limited its DNA binding ability, which affected its function as a transcriptional factor. Additionally, low levels of STAT6 in prostate cancer tissue were associated with an increased overall survival rate and a reduced biochemical relapse rate in the $\mathrm{ONCOMINE}^{\circledR}$ database.

\section{Materials and methods}

Retrieval of the ONCOMINE ${ }^{\circledR}$ database and GSE40026 and GSE21032 datasets. Microarray datasets for prostate cancer were retrieved from the ONCOMINE ${ }^{\circledR}$ Cancer Profiling Database (www.oncomine.org) to investigate STAT6 expression levels including Wallace, Grasso and Lapointe datasets. The co-expression data of miR-135a and STAT6 in prostate cancer cell lines was retrieved from the GSE40026 (10) and GSE21032 (11) datasets in the Gene Expression Omnibus database.

Gene Set Enrichment Analysis (GSEA) with STAT6 expression levels. The Cancer Genome Atlas (TCGA) and Memorial Sloan-Kettering Cancer Center (MSKCC) prostate cancer databases were used to generate STAT6 positively- and negatively-associated gene sets. The Pearson score was calculated for STAT6-correlated genes and $>+0.3$ was recognized as positively correlated while $<-0.3$ was considered negatively correlated. The pre-ranked gene sets were deposited into GSEA (12).

Cell culture and transfection. The DU145 and PC3 human prostate cancer cell lines were obtained from the American Type Culture Collection (ATCC) and grown according to the ATCC protocol. Briefly, the cell lines were cultured in Dulbecco's modified Eagle's medium or F12K medium supplemented respectively with $50 \mathrm{U} / \mathrm{ml}$ penicillin, $50 \mathrm{mg} / \mathrm{ml}$ streptomycin (Gibco; Thermo Fisher Scientific, Inc., Waltham, MA, USA) and $10 \%$ fetal bovine serum (FBS; Hangzhou Sijiqing Biological Engineering Materials Co., Ltd., Hangzhou, China) at $37^{\circ} \mathrm{C}$ in an atmosphere of $5 \% \mathrm{CO}_{2}$. Transfections with miR-135a and negative control (NC) mimics (Shanghai GenePharma Co., Ltd., Shanghai, China) were performed at a concentration of $40 \mathrm{nM}$ Lipofectamine ${ }^{\circledR} 2000$ (Invitrogen; Thermo Fisher Scientific, Inc.) according to the manufacturer's protocol.

Hoechst33342 staining. The DU145 and PC3 cells were cultured on slides in a six-well plate. Following transfection for $48 \mathrm{~h}$, the cells were washed with PBS and stained with
Hoechst33342 (1 mg/ml; Beyotime Institute of Biotechnology, Haimen, China) for $5 \mathrm{~min}$ at $37^{\circ} \mathrm{C}$. Following a second wash with PBS, images of the cells were captured and counted using immunofluorescence microscopy (Olympus Corporation, Tokyo, Japan).

Quantification of apoptosis using fluorescence-activated cell sorting (FACS). To determine the levels of apoptosis following treatment with miR-135a or NC, an Annexin-V (AV) and propidium iodide (PI; Beyotime Institute of Biotechnology) stain was performed. Briefly, DU145 and PC3 cells were harvested $72 \mathrm{~h}$ post-transfection, washed in PBS and suspended in binding buffer (Beyotime Institute of Biotechnology) and cells were serum-deprived for $24 \mathrm{~h}$ prior to FACS. Following this, the cells were stained with AV and PI $(50 \mu \mathrm{M})$ and incubated at room temperature for $15 \mathrm{~min}$. A total of 10,000 events were recorded using a flow cytometer (BD Biosciences, Franklin Lakes, NJ, USA) and the proportion of apoptotic cells was analyzed.

Western blotting. DU145 and PC3 cells were transfected with miR-135a or NC after $72 \mathrm{~h}$ prior to being washed with cold PBS and lysed in RIPA buffer (Beyotime Institute of Biotechnology) on ice. The protein concentration was determined by bicinchoninic acid protein assay kit (Beyotime Institute of Biotechnology). The western blot protocol was performed as described in previous studies $(13,14)$. Proteins were probed with anti-STAT6 (catalog no. 9362, dilution, 1:500), anti-phosphorylated (p)-STAT6 (Tyr641) (catalog no. 9361, dilution, 1:500) and anti-GAPDH (catalog no. 2118, dilution, 1:2,000) (Cell Signaling Technology, Inc., Danvers, MA, USA). The expression of p-STAT6 was induced by IL-4 (100 ng/ml).

Electrophoretic mobility shift assay (EMSA). An EMSA was conducted to evaluate the DNA-binding activity of STAT6 in miR-135a-transfected or NC-transfected cells. Briefly, following transfection of DU145 and PC3 cells for $72 \mathrm{~h}$, nuclear proteins from each sample were extracted with Nuclear Extraction kit (Merck KGaA, Darmstadt, Germany) and subjected to EMSA following the manufacturer's standard protocol using the LightShift ${ }^{\circledR}$ Chemiluminescent EMSA kit (Thermo Fisher Scientific, Inc.). The STAT6 target probe was synthesized with a 3 '-biotin modification (Invitrogen; Thermo Fisher Scientific, Inc.) and the sequence was 5'-acgttg aatTTCTAAGAAagaggga-3', in which the binding region is underlined.

Luciferase assay. The 3'UTR of STAT6 was amplified using polymerase chain reaction and the primer sequences were as follows: Sense, 5'-CCGACGCGTTCTTTTCTGTTGCCC CATC-3'; and antisense, 5'-CCCAAGCTTGGACCGCAG CACCTATCT-3'. The fragment was cloned downstream of the luciferase gene in a pMIR-REPORT ${ }^{\mathrm{TM}}$ Luciferase miRNA Expression Reporter vector (Ambion ${ }^{\circledR}$; Thermo Fisher Scientific, Inc.) The pMIR-Luciferase NC vector or gene-specific vector was co-transfected with miR-135a or NC into DU145 and PC3 cells. pRL Renilla luciferase control reporter vector (Promega Corporation, Madison, WI, USA) was also transfected with each condition as a reference control with Lipofectamine ${ }^{\circledR} 2000$ (Invitrogen; Thermo Fisher 
A

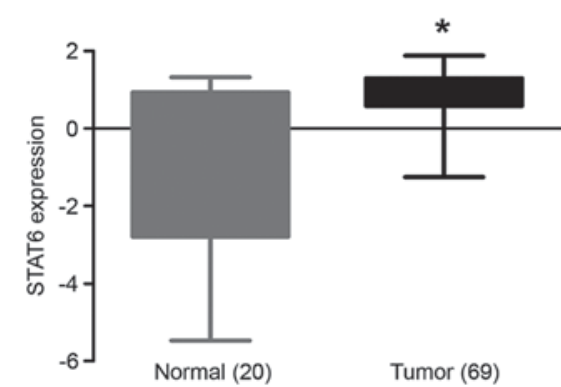

C

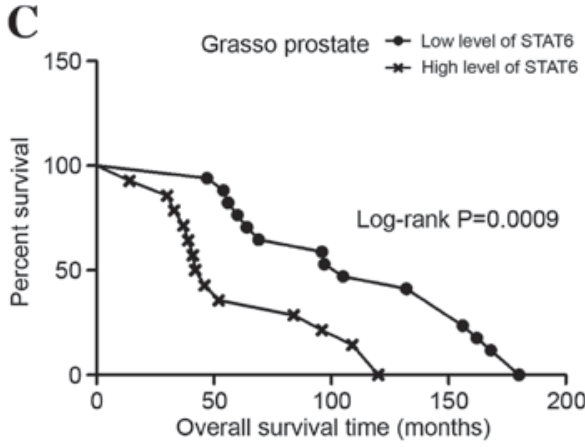

$\mathbf{E}$
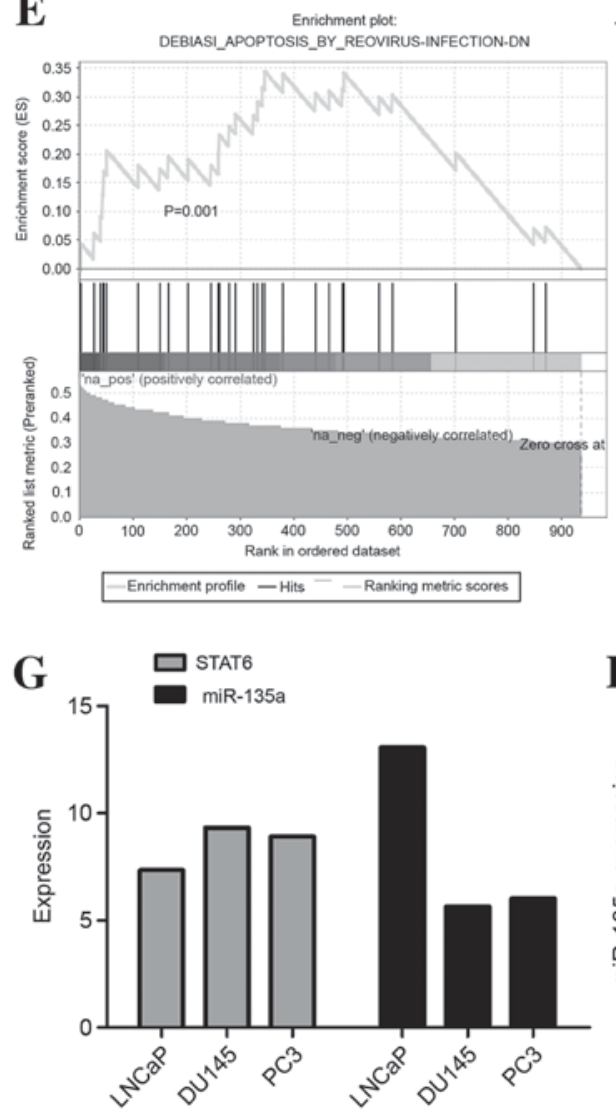

B

TCGA prostate

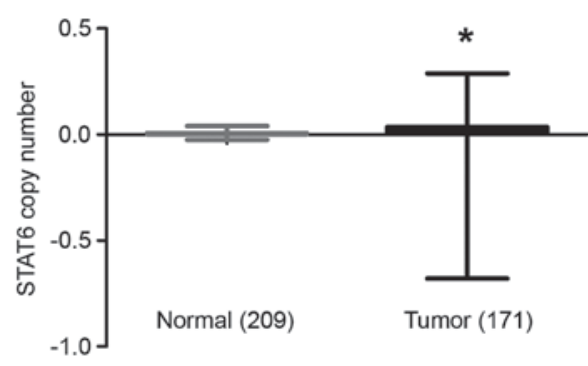

D

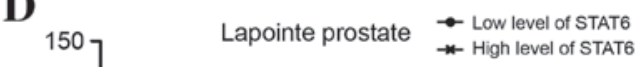

F

Enrichment plot
SHEDDEN_LUNG_CANCER_POOR_SURVIVAL_A6

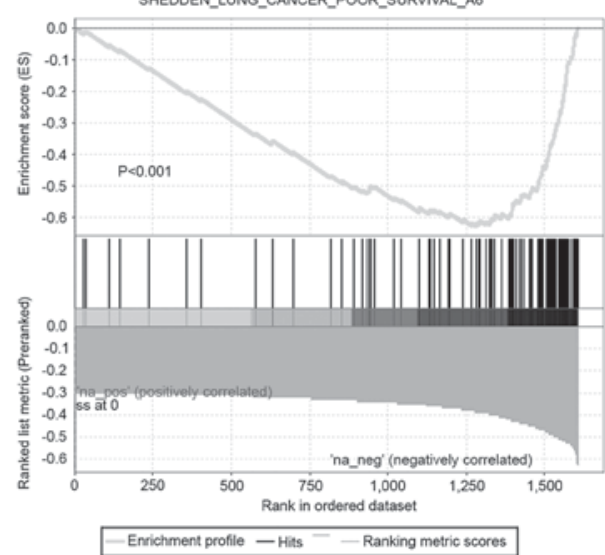

H

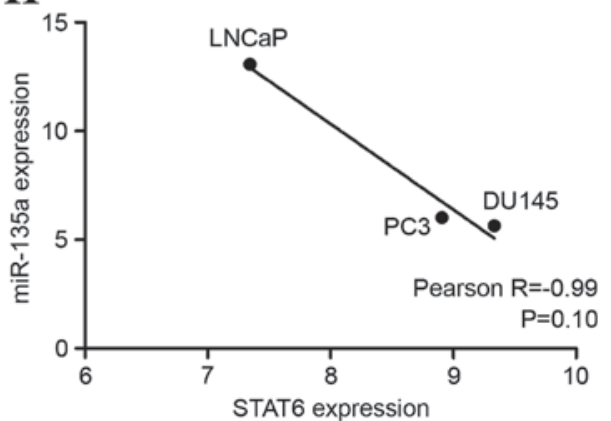

Figure 1. ONCOMINE ${ }^{\circledast}$ prostate cancer database analyses. (A) STAT6 was overexpressed in prostate cancer tissues compared with normal prostate gland tissues in the Wallace study ( $\left.{ }^{*} \mathrm{P}<0.0001\right)$. (B) The tumors in the TCGA dataset have an increased STAT6 copy number ("P=0.0002). (C) Increased levels of STAT6 expression were associated with a reduced overall survival rate in the Grasso study $(\mathrm{P}=0.0009)$. (D) Increased levels of STAT6 expression were associated with the biochemical relapse rate $(\mathrm{P}=0.10)$ following radical prostatectomy in the Lapointe study. (E and $\mathrm{F})$ GSEA analyses of gene ontology terms indicated that there was enriched expression of gene sets involved in $(\mathrm{E})$ cell apoptosis $(\mathrm{P}=0.001)$ and $(\mathrm{F})$ cancer survival $(\mathrm{P}<0.001)$. ( $\mathrm{G}$ and $\mathrm{H})$ Microarray data from the GSE40026 and GSE21032 datasets were retrieved and revealed that miR-135a was underexpressed, whereas STAT6 was overexpressed in DU145 and PC3 cells compared with LNCaP cells ( $\mathrm{P}=0.10)$. STAT6, signal transducer and activator or transcription 6; TCGA, The Cancer Genome Atlas; GSEA, Gene Set Enrichment Analysis; GSEA, Gene Series; miR-135a, microRNA-135a.

Scientific, Inc.). A total of $72 \mathrm{~h}$ after transfection, the firefly luciferase and Renilla luciferase activities were measured using the Dual-Luciferase ${ }^{\circledR}$ Reporter assay system (Promega Corporation). 

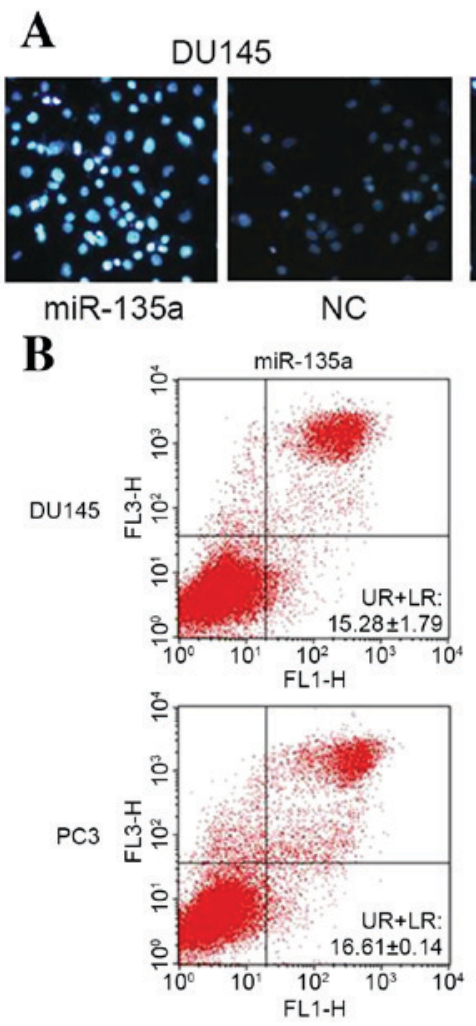

PC3

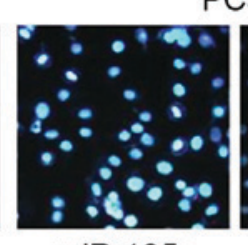

miR-135a
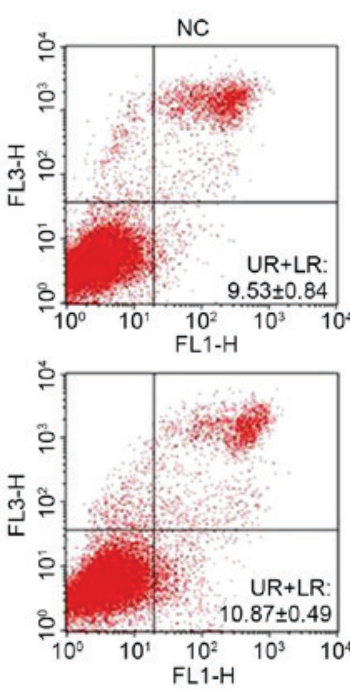

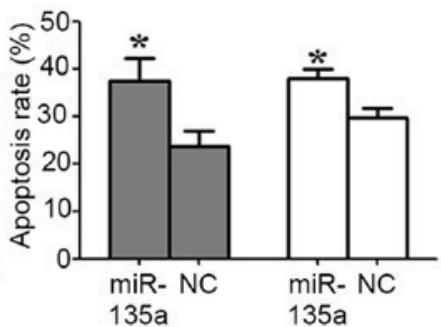

DU145

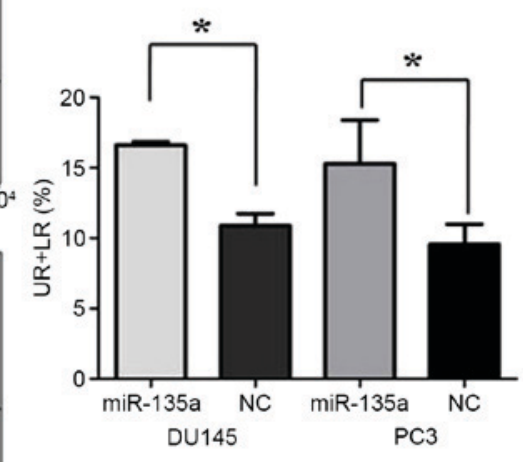

Figure 2. miR-135a induced prostate cancer cell apoptosis in vitro. (A) miR-135a induced prostate cancer cell apoptosis, as detected using a Hoechst stain assay. ${ }^{*} \mathrm{P}<0.05$ vs. the NC group. (B) The effect of miR-135a on apoptosis was confirmed using FACS. Data presents the mean \pm standard deviation of $>3$ independent experiments. ${ }^{*} \mathrm{P}<0.05$. miR-135a, microRNA-135a; FACS, fluorescence-activated cell sorting; NC, negative control; UR, upper right quadrant; LR, lower right quadrant; FL-H, fluorescence-height.

Bioinformatics and statistical analysis. The online miRNA databases TargetScan 5.1 (15) and RNAhybrid (16) were used to determine the predicted miR-135a target genes. Statistical analyses were performed using SPSS software version 16.0 (SPSS, Inc., Chicago, IL, USA). The aforementioned experiments were repeated three times. Data were presented as the mean \pm standard deviation. Group means were compared using the Student's t-test. Survival data was analyzed using the Kaplan-Meier estimate and $\log$-rank P was calculated. $\mathrm{P}<0.05$ was considered to indicate a statistically significant difference.

\section{Results}

STAT6 expression levels and its prognostic role in prostate cancer in the ONCOMINE ${ }^{\circledR}$ prostate database and co-expression of miR-135a and STAT6 in prostate cancer cell lines. The ONCOMINE ${ }^{\circledR}$ prostate cancer database is an established database containing several high-quality datasets. In the Wallace prostate cancer dataset, STAT6 expression levels in tumor tissues were higher compared with those in normal prostate gland tissues $(\mathrm{P}<0.0001$; Fig. 1A) and tumors exhibited a higher STAT6 copy number in the TCGA dataset $(\mathrm{P}=0.0002$; Fig. 1B). The Grasso and Lapointe prostate cancer datasets contain data on the overall survival time of patients with metastatic cancer and the biochemical relapse-free survival time, which are important events in the progression of prostate cancer. An increased level of STAT6 expression was associated with a significantly reduced overall survival rate in Grasso's study ( $\mathrm{P}=0.0009$; Fig. 1C) and also an increased biochemical relapse rate $(\mathrm{P}=0.1000$; Fig. 1D) following radical prostatectomy in Lapointe's study, but this was not statistically significant. Additionally, in the TCGA and MSKCC databases, STAT6-correlated genes were involved in cell apoptosis signaling pathways $(\mathrm{P}=0.001$; Fig. $1 \mathrm{E})$ and cancer survival $(\mathrm{P}<0.001$; Fig. $1 \mathrm{~F})$.

To investigate the co-expression of miR-135a and STAT6 in DU145, PC3 and LNCaP prostate cancer cell lines, microarray data from the GSE40026 and GSE21032 datasets were evaluated and the results revealed that miR-135a was underexpressed and STAT6 was overexpressed in DU145 and PC3 cells compared with LNCaP cells. This indicated an inverse correlation between miR-135a and STAT6 expression in these cell lines $(\mathrm{P}=0.10$; Fig. $1 \mathrm{G}$ and $\mathrm{H})$.

miR-135a increases apoptosis in prostate cancer cells. To determine whether the overexpression of miR-135a affects the levels of apoptosis in prostate cancer cells, Hoechst staining and FACS analyses were performed. Notably, overexpression of miR-135a in DU145 and PC3 cells led to an increase in the percentage of Hoechst-positive cells $(37.3 \pm 2.7$ vs. $23.67 \pm 1.9 \%$ in DU145 cells and $38.0 \pm 1.2$ vs. $29.7 \pm 1.2 \%$ in PC3 cells; $\mathrm{P}=0.0144$ in DU145 and $\mathrm{P}=0.0075$ in PC3; Fig. 2A). To further examine this result, the effects of miR-135a on the levels of apoptosis were investigated using FACS analysis. DU145 and PC3 cells were transfected with miR-135a or NC and cells were serum-deprived for $24 \mathrm{~h}$ prior to FACS. Compared with NC-transfected cells, the proportion of apoptotic cells 

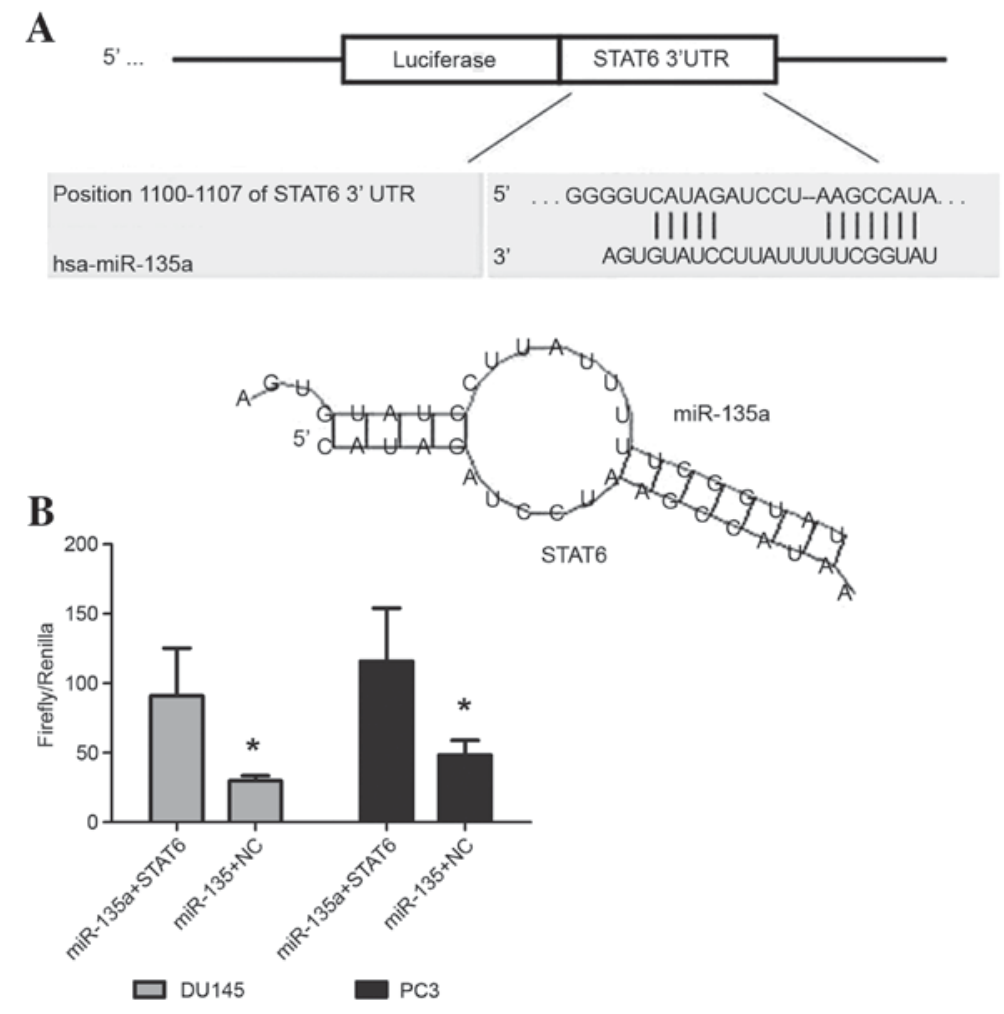

Figure 3. STAT6 is a direct target of miR-135a in prostate cancer cells. (A) TargetScan and RNAhybrid databases revealed that miR-135a may bind to a target sequence located in the region of nucleotides 1,100-1,107 of the 3'UTR of STAT6. (B) The STAT6 3'UTR was cloned into a luciferase reporter cloning site in the pMIR-report ${ }^{\mathrm{TM}}$ Luciferase miRNA Expression Reporter vector and increased miR-135a level was associated with decreased luciferase activity. "P<0.05. STAT6, signal transducer and activator or transcription 6; miR-135a, microRNA-135a; UTR, untranslated region; miRNA, microRNA; NC, negative control.
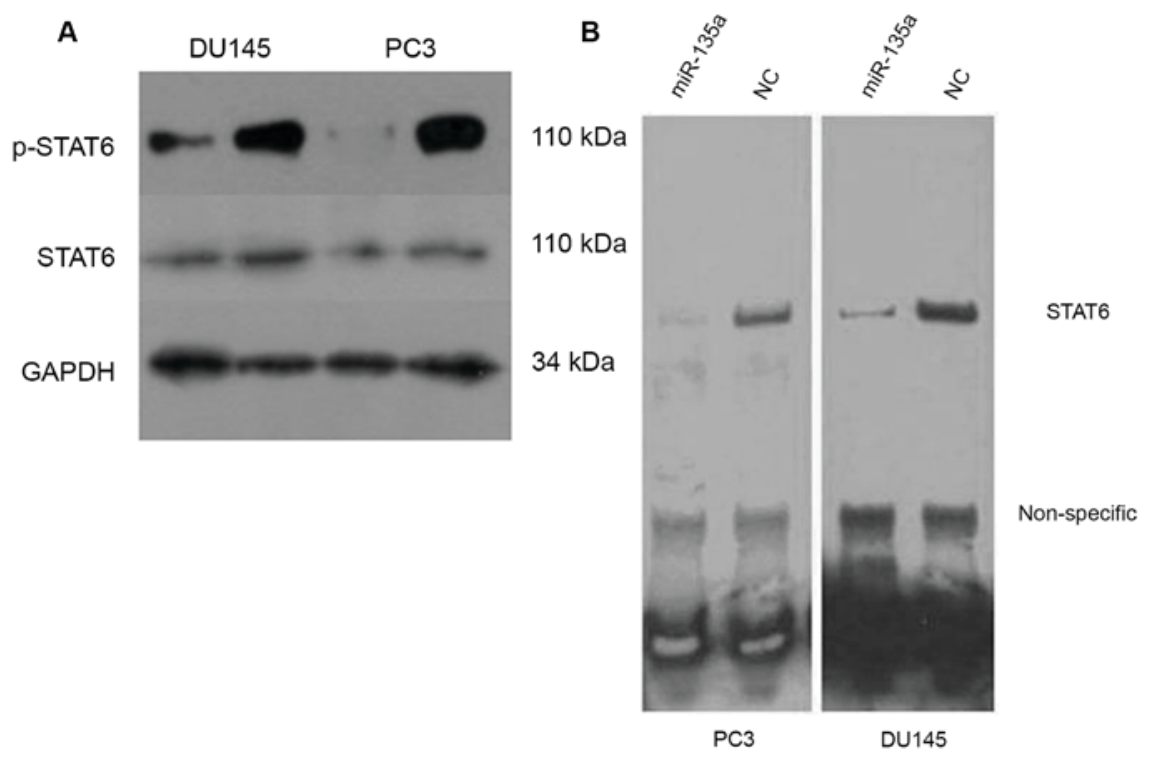

Figure 4. Protein expression levels and DNA-binding ability of STAT6 and p-STAT6 in prostate cancer cells treated with miR-135a and NC. (A) Endogenous STAT6 and p-STAT6 stimulated with IL-4 were inhibited by transfection with miR-135a in western blot analysis. (B) An electrophoretic mobility shift assay was performed to assess the binding of a consensus STAT6 DNA oligo probe using miR-135a and NC nuclear extracts and indicated a decrease in DNA-binding activity in prostate cancer cells following induced miR-135a expression. STAT6, signal transducer and activator or transcription 6; p-STAT6, phosphorylated signal transducer and activator or transcription 6; miR-135a, microRNA-135a; IL-4, interleukin 4; NC, negative control.

following transfection with miR-135a was significantly increased $(15.28 \pm 1.79$ vs. $9.53 \pm 0.84 \%$ in DU145 cells and $16.61 \pm 0.14$ vs. $10.87 \pm 0.49 \%$ in $\mathrm{PC} 3$ cells; $\mathrm{P}=0.0073$ in DU145 and $\mathrm{P}<0.0001$ in PC3; Fig. 2B).
STAT6 is a direct target of miR-135a. It is established that miRNAs exert their function by directly binding to target genes. Therefore, STAT6 was selected using TargetScan 5.1 (15) and RNAhybrid (8). To investigate whether STAT6 
is a direct target of miR-135a, the predicted binding site $(1,100-1,107 \mathrm{bp})$ of miR-135a was cloned into the STAT6 3'UTR vector (Fig. 3A). The luciferase reporter expression was significantly reduced following co-transfection with miR-135a compared with the NC group (Fig. 3B).

miR-135a negatively regulated the protein expression and the function of STAT6. Western blot analyses for STAT6 expression in the cells transfected with miR-135a or NC demonstrated that endogenous STAT6 protein levels were downregulated in miR-135a-transfected prostate cancer cells (Fig. 4A). STAT6 is able to function as a transcriptional factor following its phosphorylation (17), therefore p-STAT6 expression levels and DNA-binding ability were analyzed using western blotting and the EMSA assay. The results revealed that the expression levels of p-STAT6 were also reduced by miR-135a transfection following treatment with interleukin-4 (IL-4; $100 \mathrm{ng} / \mathrm{ml}$ ). Additionally, EMSA demonstrated that the STAT6 DNA-binding activity in prostate cancer cells was inhibited via the overexpression of miR-135a (Fig. 4B).

\section{Discussion}

Elucidating the precise molecular mechanisms underlying tumorigenesis is important for the development of improved therapy strategies for patients with prostate cancer, particularly for patients with metastases. Previous studies have suggested that the dysregulation of miRNA is involved in the formation of metastases, including miR-221/-222 (18) and miR-146a (14). The preliminary bioinformatic analyses in the current study focused on miR-135a. The expression levels of miR-135a in cancer are controversial, as it has been reported to be upregulated and downregulated in distinct tumors and may function as a tumor-inducer in colorectal (19) and breast cancer (20) and a tumor-suppressor in renal-cell carcinoma (21) and gastric cancer (22). Additionally, the overexpression of miR-135a sensitized lung cancer cells to cisplatin (23), whereas suppression of miR-135a sensitized drug-resistant cell lines to paclitaxel-induced cell death (24). Prostate cancer has a distinct progression and the underlying mechanisms are yet to be elucidated. The aberrant expression, subsequent function and underlying mechanisms of miR-135a in prostate cancer are yet to be established. In the present study, STAT6 inhibition via miR-135a promoted apoptosis in prostate cancer cells and it was demonstrated that STAT6 is a constitutively expressed survival factor in several human prostate cancer datasets, and may a direct functional target of miR-135a.

Prior to activation, STAT family proteins are cytosolic and, following activation, translocate into the nucleus and function as transcription factors. Sustained STAT activity has been described in a number of tumor types (25). STAT6 serves an important role in exerting IL-4-mediated biological responses. Following stimulation by IL-4, STAT6 is phosphorylated via receptor-associated kinases and translocated into the cell nucleus (25). Das et al (5) demonstrated that STAT6 expression levels were significantly correlated with higher histological grades of prostate cancer and with tumor size. Downregulation of STAT6 using siRNA promoted the induction of apoptosis and a reduction in cell viability in
DU145 cells. Due to the established roles of STAT6 in prostate cancer, the present study investigated the role of STAT6 and its association with miR-135a. Concordant with previous studies, the results provided novel data to support the hypothesis that the downregulation of STAT6 in prostate cancer may occur as a result of the overexpression of the miRNA molecule miR-135a, which may indicate a regulatory mechanism that is able to drive apoptosis in prostate cancer cells.

In conclusion, miR-135a was identified as an important miRNA that may regulate the levels of apoptosis in prostate cancer cells. It was hypothesized that the miR-135a-STAT6 association may be a novel target for prognostic and therapeutic advances in prostate cancer.

\section{Acknowledgements}

Not applicable.

\section{Funding}

The present study was supported by the National Natural Science Foundation of China (grant no. 81202034), the Science Foundation of Ministry of Education of China (grant no. 20120092120071) and the Natural Science Foundation of Jiangsu Province (grant nos. BK2012336 and BL2013032), Nanjing City (grant no. 201201053) and the Southeast University (grant no. 3290002402).

\section{Availability of data and materials}

The datasets used and/or analyzed during the current study are available from the corresponding author on reasonable request.

\section{Authors' contributions}

BX and MC participated in study design and drafted the manuscript. XL and YZ participated in collection of data and manuscript preparation. CL and XH performed the statistical analysis and participated in the critical revision of the manuscript. SC, WZ and LZ assisted in data analysis, interpretation of the data and critically revised the manuscript for important intellectual content. All authors read and approved the final manuscript.

\section{Ethics approval and consent to participate}

Not applicable.

\section{Patient consent for publication}

Not applicable.

\section{Competing interests}

The authors declare that they have no competing interests.

\section{References}

1. Siegel R, Naishadham D and Jemal A: Cancer statistics, 2013. CA Cancer J Clin 63: 11-30, 2013. 
2. Feldman BJ and Feldman D: The development of androgen-independent prostate cancer. Nat Rev Cancer 1: 34-45, 2001.

3. Debatin KM: Apoptosis pathways in cancer and cancer therapy. Cancer Immunol Immunother 53: 153-159, 2004.

4. Tran C, Ouk S, Clegg NJ, Chen Y, Watson PA, Arora V, Wongvipat J, Smith-Jones PM, Yoo D, Kwon A, et al: Development of a second-generation antiandrogen for treatment of advanced prostate cancer. Science 324: 787-790, 2009.

5. Das S, Roth CP, Wasson LM and Vishwanatha JK: Signal transducer and activator of transcription-6 (STAT6) is a constitutively expressed survival factor in human prostate cancer. Prostate 67: 1550-1564, 2007.

6. Kotb S, Mosharafa A, Essawi M, Hassan H, Meshref A and Morsy A: Circulating miRNAs 21 and 221 as biomarkers for early diagnosis of prostate cancer. Tumour Biol 35: 12613-12617, 2014.

7. Chang YL, Zhou PJ, Wei L, Li W, Ji Z, Fang YX and Gao WQ: MicroRNA-7 inhibits the stemness of prostate cancer stem-like cells and tumorigenesis by repressing KLF4/PI3K/Akt/p21 pathway. Oncotarget 6: 24017-24031, 2015.

8. Kojima S, Enokida H, Yoshino H, Itesako T, Chiyomaru T, Kinoshita T, Fuse M, Nishikawa R, Goto Y, Naya Y, et al: The tumor-suppressive microRNA-143/145 cluster inhibits cell migration and invasion by targeting GOLM1 in prostate cancer. J Hum Genet 59: 78-87, 2014

9. Sun Q, Zhao X, Liu X, Wang Y, Huang J, Jiang B, Chen Q and Yu J: miR-146a functions as a tumor suppressor in prostate cancer by targeting Rac1. Prostate 74: 1613-1621, 2014.

10. Kobayashi N, Uemura H, Nagahama K, Okudela K, Furuya M, Ino Y, Ito Y, Hirano H, Inayama Y, Aoki I, et al: Identification of miR-30d as a novel prognostic maker of prostate cancer. Oncotarget 3: 1455-1471, 2012.

11. Taylor BS, Schultz N, Hieronymus H, Gopalan A, Xiao Y, Carver BS, Arora VK, Kaushik P, Cerami E, Reva B, et al: Integrative genomic profiling of human prostate cancer. Cancer Cell 18: 11-22, 2010

12. Mootha VK, Lindgren CM, Eriksson KF, Subramanian A, Sihag S, Lehar J, Puigserver P, Carlsson E, Ridderstråle M, Laurila E, et al: PGC-1alpha-responsive genes involved in oxidative phosphorylation are coordinately downregulated in human diabetes. Nat Genet 34: 267-273, 2003.

13. Xu B, Niu X, Zhang X, Tao J, Wu D, Wang Z, Li P, Zhang W, Wu H, Feng N, et al: miR-143 decreases prostate cancer cells proliferation and migration and enhances their sensitivity to docetaxel through suppression of KRAS. Mol Cell Biochem 350: 207-213, 2011.

14. Xu B, Wang N, Wang X, Tong N, Shao N, Tao J, Li P, Niu X, Feng N, Zhang L, et al: MiR-146a suppresses tumor growth and progression by targeting EGFR pathway and in a p-ERK-dependent manner in castration-resistant prostate cancer. Prostate 72: $1171-1178,2012$
15. Garcia DM, Baek D, Shin C, Bell GW, Grimson A and Bartel DP: Weak seed-pairing stability and high target-site abundance decrease the proficiency of 1sy- 6 and other microRNAs. Nat Struct Mol Biol 18: 1139-1146, 2011.

16. Krüger J and Rehmsmeier M: RNAhybrid: microRNA target prediction easy, fast and flexible. Nucleic Acids Res 34 (Web Server Issue): W451-W454, 2006.

17. Rahal OM, Wolfe AR, Mandal PK, Larson R, Tin S, Jimenez C, Zhang D, Horton J, Reuben JM, McMurray JS and Woodward WA Blocking interleukin (IL)4- and IL13-mediated phosphorylation of STAT6 (Tyr641) decreases M2 polarization of macrophages and protects against macrophage-mediated radioresistance of inflammatory breast cancer. Int J Radiat Oncol Biol Phys 100: 1034-1043, 2018

18. Sun T, Wang X, He HH, Sweeney CJ, Liu SX, Brown M, Balk S, Lee GS and Kantoff PW: MiR-221 promotes the development of androgen independence in prostate cancer cells via downregulation of HECTD2 and RAB1A. Oncogene 33: 2790-2800, 2014

19. Zhou W, Li X, Liu F, Xiao Z, He M, Shen S and Liu S: MiR-135a promotes growth and invasion of colorectal cancer via metastasis suppressor 1 in vitro. Acta Biochim Biophys Sin (Shanghai) 44: 838-846, 2012.

20. Chen Y, Zhang J, Wang H, Zhao J, Xu C, Du Y, Luo X, Zheng F, Liu R, Zhang $\mathrm{H}$ and Ma D: miRNA-135a promotes breast cancer cell migration and invasion by targeting HOXA10. BMC Cancer 12: 111, 2012.

21. Yamada Y, Hidaka H, Seki N, Yoshino H, Yamasaki T, Itesako T, Nakagawa $\mathrm{M}$ and Enokida $\mathrm{H}$ : Tumor-suppressive microRNA-135a inhibits cancer cell proliferation by targeting the c-MYC oncogene in renal cell carcinoma. Cancer Sci 104: 304-312, 2013.

22. Wu H, Huang M, Cao P, Wang T, Shu Y and Liu P: MiR-135a targets JAK2 and inhibits gastric cancer cell proliferation. Cancer Biol Ther 13: 281-288, 2012.

23. Zhou L, Qiu T, Xu J, Wang T, Wang J, Zhou X, Huang Z, Zhu W, Shu Y and Liu P: miR-135a/b modulate cisplatin resistance of human lung cancer cell line by targeting MCL1. Pathol Oncol Res 19: 677-683, 2013.

24. Holleman A, Chung I, Olsen RR, Kwak B, Mizokami A, Saijo N, Parissenti A, Duan Z, Voest EE and Zetter BR: miR-135a contributes to paclitaxel resistance in tumor cells both in vitro and in vivo. Oncogene 30: 4386-4398, 2011.

25. Watson CJ: Stat transcription factors in mammary gland development and tumorigenesis. J Mammary Gland Biol Neoplasia 6: 115-127, 2001. 